\title{
EFICÁCIA CLÍNICA DO LASER ACUPUNTURAL NO TRATAMENTO DA DESORDEM TEMPOROMANDIBULAR
}

\author{
Diego Rodrigues Pessoa ${ }^{1}$ \\ Davidson Ribeiro Costa ${ }^{2}$ \\ Bruno de Moraes Prianti ${ }^{3}$ \\ David Ribeiro Costa ${ }^{4}$ \\ Carolina Alves Delpasso ${ }^{5}$ \\ Vanessa Borelli Seefeldt ${ }^{6}$ \\ Brenda Barros Magalhães ${ }^{7}$ \\ Simone Isidoro Gandine ${ }^{8}$ \\ Renata Amadei Nicolau ${ }^{9}$
}

Resumo: A disfunção temporomandibular (DTM) é a condição mais frequente de dor orofacial crônica. A DTM é caracterizada pela a presença de sinais e sintomas na região orofacial. A acupuntura e Terapia a laser de baixa intensidade (TLBI) vêm sendo empregadas no tratamento da DTM. A acupuntura consiste na inserção de agulhas em acupontos específicos do corpo para promover efeitos terapêuticos. A TLBI é uma modalidade de tratamento não invasiva, que vem sendo utilizada com frequência na prática clínica para o tratamento das DTM. Diante do exposto, o objetivo deste estudo foi realizar revisão de literatura, acerca da eficácia clinica do laser acupuntural em pacientes com a DTM. O emprego do laseracupuntural no tratamento das DTM favorece o controle antálgico, miorrelaxante e anti-inflamatório, no entanto, são necessárias novas pesquisas acerca do tema para determinar a eficácia do laser acupuntural em longo prazo para o tratamento de desordens temporomandibulares.

Palavras-chave: Low level therapy; Acupuncture therapy; Physical therapy modalities.

\footnotetext{
1 Centro de Laserterapia e Fotobiologia - CELAFO, Instituto de Pesquisa e Desenvolvimento/Universidade do Vale do Paraíba, Brasil. E-mail: fisio.diegorodrigues@gmail.com.

2 Centro de Laserterapia e Fotobiologia - CELAFO, Instituto de Pesquisa e Desenvolvimento/Universidade do Vale do Paraíba, Brasil. E-mail: dnribcosta@hotmail.com.

3 Centro de Laserterapia e Fotobiologia - CELAFO, Instituto de Pesquisa e Desenvolvimento/Universidade do Vale do Paraíba, Brasil. E-mail: brunoprianti@hotmail.com.

${ }^{4}$ Centro de Laserterapia e Fotobiologia - CELAFO, Instituto de Pesquisa e Desenvolvimento/Universidade do Vale do Paraíba, Brasil. E-mail: profdavid8@gmail.com.

${ }^{5}$ Centro de Laserterapia e Fotobiologia - CELAFO, Instituto de Pesquisa e Desenvolvimento/Universidade do Vale do Paraíba, Brasil. E-mail: delpassoc@gmail.com.

${ }^{6}$ Centro de Laserterapia e Fotobiologia - CELAFO, Instituto de Pesquisa e Desenvolvimento/Universidade do Vale do Paraíba, Brasil. E-mail: neborelli@yahoo.com.br.

7 Centro de Laserterapia e Fotobiologia - CELAFO, Instituto de Pesquisa e Desenvolvimento/Universidade do Vale do Paraíba, Brasil. E-mail: bre.barros@outlook.com.

${ }^{8}$ Centro de Laserterapia e Fotobiologia - CELAFO, Instituto de Pesquisa e Desenvolvimento/Universidade do Vale do Paraíba, Brasil. E-mail: simonegandine@hotmail.com.

${ }^{9}$ Centro de Laserterapia e Fotobiologia - CELAFO, Instituto de Pesquisa e Desenvolvimento/Universidade do Vale do Paraíba, Brasil. E-mail: renatanicolau@hotmail.com.
} 